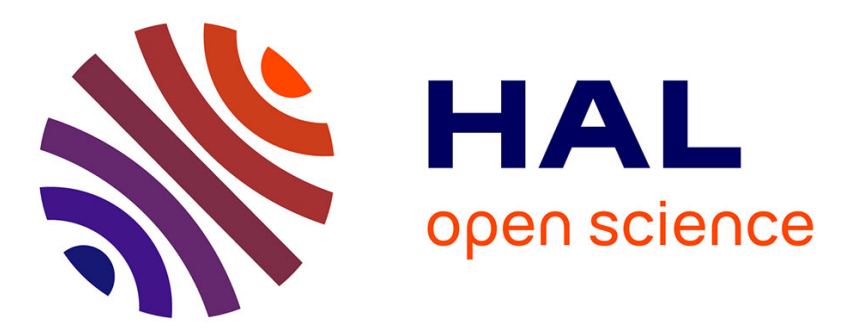

\title{
Simulating Physical Interactions between an Articulated Mobile Vehicle and a Terrain
}

\author{
Stéphane Jimenez, Annie Luciani, Christian Laugier
}

\section{To cite this version:}

Stéphane Jimenez, Annie Luciani, Christian Laugier. Simulating Physical Interactions between an Articulated Mobile Vehicle and a Terrain. ORIA'91, 1991, Marseille, France. pp.11. hal-00910550

\section{HAL Id: hal-00910550 \\ https://hal.science/hal-00910550}

Submitted on 11 Mar 2014

HAL is a multi-disciplinary open access archive for the deposit and dissemination of scientific research documents, whether they are published or not. The documents may come from teaching and research institutions in France or abroad, or from public or private research centers.
L'archive ouverte pluridisciplinaire HAL, est destinée au dépôt et à la diffusion de documents scientifiques de niveau recherche, publiés ou non, émanant des établissements d'enseignement et de recherche français ou étrangers, des laboratoires publics ou privés. 


\title{
Simulating physical interactions between an articulated mobile vehicle and a terrain
}

\author{
Stéphane Jimenez, Annie Luciani \\ ACROE-IMAG/LIFIA , 46 Av. Félix Viallet, 38031 Grenoble Cedex \\ e-mail : acroe@lifia.imag.fr \\ Christian Laugier \\ IRIMAG-IMAG/LIFIA, 46 Av. Félix Viallet, 38031 Grenoble Cedex \\ e-mail : laugier@lifia.imag.fr
}

\begin{abstract}
This paper deals with the problem of simulating the motions of a complex land vehicle moving in a natural environment. The contribution presented here is a motion generator which predicts the dynamic behaviour of the vehicle when executing a given nominal motion plan. This plan is expressed in terms of a channel to follow and of a set of intermediate subgoals to reach. Solving this motion generation problem requires to explicitly reason about the geometric and the physical aspects of the movements that the vehicle has to execute. In our approach, this is done using two basic constructions derived from the concept of physical model: the "generalized obstacles" are used for physically guiding the movements of the vehicle using an explicit model of the vehicle/terrain interactions, and the "physical targets" are used to map the strategic information onto our physical representation of the world and to introduce the force feedback gestural control.
\end{abstract}

\section{Introduction}

This paper deals with the problem of simulating the motions of a complex land vehicle moving in a natural environment containing both obstacles that have to be avoided and obstacles that can be crossed over by the vehicle. The framework of this research program is the VAP project conducted by the CNES (French spatial agency) in relation with several French Research Institutes (CNRS, INRIA, CEA, CERT). The purpose of this project is to design a complete system for teleprogramming an autonomous planetary rover.

The contribution presented in this paper is a motion generator which predicts the dynamic behaviour of a land vehicle when executing a given nominal motion plan. This plan is supposed to be provided by a geometric path planner. It is expressed in terms of a channel to follow and of a set of intermediate subgoals to reach. Solving this motion generation problem requires to explicitly reason about the geometric and the physical aspects of the movements that the vehicle has to execute.

Until now, very few methods have been devised for dealing with the effects of dynamic in motion planning. Some researchers have designed adapted architectures for controlling in a 
dynamic way the locomotion of several types of mobile robots (legged robots for instance [13]), without trying to solve the associated motion planning problem. Because of the intrinsic complexity of this problem, most of the work done on this topic has led to the implementation of various geometric path planners (the considered subproblem is to find a collision-free trajectory for the robot) [9]. More recent methods have been devised for the purpose of dealing with non integrable kinematic constraints when generating such paths (see [1], [6] and [10]). Despite the great ability of the previous planners to solve such geometric based problems, the automatic generation of executable safe motions for a land vehicle moving in a natural environment is beyond the current capabilities of these planners (because the physical vehicle/terrain interactions cannot be processed using purely geometric and kinematic models). Since such interactions are of a major importance in this context -because friction, sliding and skid phenomena may strongly modify the behaviour of the vehicle-, it is necessary to make use of appropriate physical models for predicting the robot behaviour.

This is why we have decided to combine a geometric path planner with a motion generator having the ability of simulating physical interactions. This approach is described in $\& 2$. It basically consists in combining strategic and tactical information through two complementary concepts (see \&4): the concept of generalized obstacle which is used for physically guiding the vehicle using an explicit model of the vehicle/terrain interactions, and the concept of physical target which allows us to map the strategic information (intermediate subgoals) onto our physical representation of the world. These concepts have been implemented using physical based models first developed in the field of computer graphics and of man/machine communication [8] [11]. As shown in \&3, such a type of model can be built by combining a set of representative punctual masses within an interaction network including linear and non-linear terms. In \& 5 we explain how this new approach can lead towards a powerfull extention of the "Predictive Display" to a realistic reconstruction of the natural piloting conditions by mean of adapted force feedback devices. Several types of vehicle and of vehicle/terrain interactions have been modelized and simulated using this approach (see \&6).

\section{Outline of the approach}

As previously mentionned, our approach for planning executable safe motions for a land vehicle moving in a natural environment is to combine a geometric path planner with a motion generator having the ability of simulating physical interactions. The geometric path planner is in charge of generating a potentially safe path for the rover -i.e. a set of collision-free trajectories-. Such a path is represented by a safe channel $C$ and by a set $\left(g_{1}, g_{2} \ldots g_{n}\right)$ of intermediate subgoals. It defines a strategic information that the motion generator will use to produce the sequence of movements which results from the combined effects of the applied motion commands and of the vehicle/terrain interactions (figure 1 illustrates). Such an approach consisting in combining a global path planner with a method aimed at adapting the robot movements to the local environment of the task has already been used by some researchers for implementing geometric path planners and reactive motion controllers (see [7]). But the used models -mainly geometric representations and potential field functions- do not include the basic information which is required for processing physical interactions.

Let $M$ be the land vehicle, let $T$ and $O$ be respectively the terrain and the obstacles to avoid, and let $S P=\left(C,\left\{g_{1}, g_{2} \ldots g_{n}\right\}\right)$ be the potentially safe path generated by the geometric path planner. We will respectively denote by $G(W)$ and by $\Phi(W)$ the geometric model and the physical model which is associated to an object (or a set of objects) $W$. The geometric path planner reasons about the model $G(M, T, O)$ and the motion generator operates on the 
a)

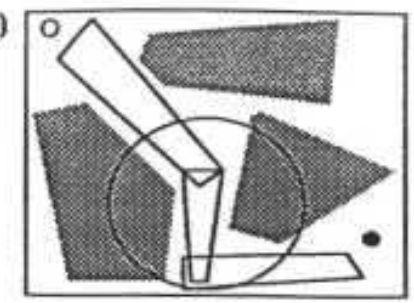

- Initial and final position of the robot

c)

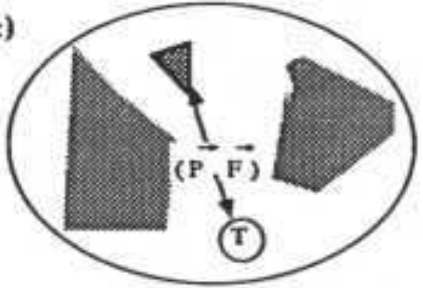

b)

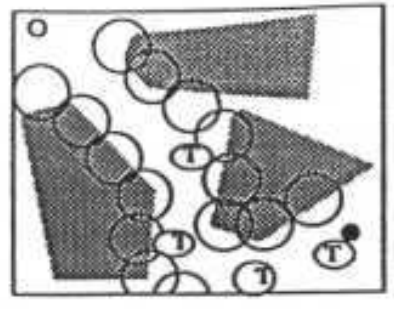

(T) Physical target (punctual mass)

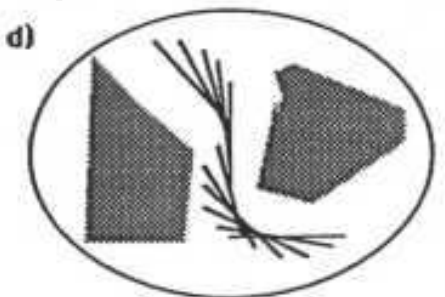

Figure 1: Illustration of our approach: (a) a selected safe channel; (b) an associated physical representation; (c) a physical target attracting the vehicle; (d) a motion resulting from the combined effects of the physical target and of the vehicle/terrain interactions.

model $\Phi(M, T, S P)$. This means that $G(M)$ and $G(T)$ have to be converted into a physical representation $\Phi(M, T)$ including an explicit model of the vehicle/terrain interactions (see \&3). This also means that appropriate mecanisms have to be developed in order to map the geometric information contained in $S P$ onto $\Phi(M, T)$. As explained in \&4, this is done using two basic constructions: $\Phi(T)$ and $\Phi(C)$ are combined within an unified model $\Phi(T \oplus C)$ called the "generalized obstacle model", and each $g_{i}$ is converted into an attractive punctual mass $\Phi\left(g_{i}\right)$ called "physical target". Using this approach, $\Phi(T \oplus C)$ can be used for physically guiding $M$, and the set $\left\{\Phi\left(g_{1}\right), \Phi\left(g_{2}\right) \ldots \Phi\left(g_{n}\right)\right\}$ can be used for representating the sequence of motion commands to apply to $M$. This approach differs from the potential field methods by the fact that it operates at a dynamic layer -i.e. any motion is directly connected by physical laws to the forces which result from objects interactions, see \&3-.

Since no complete connection has been implemented yet between the geometric path planner and the motion generator, the models $\Phi(T \oplus C)$ and $\left\{\Phi\left(g_{1}\right), \Phi\left(g_{2}\right) \ldots \Phi\left(g_{n}\right)\right\}$ are currently constructed by the operator using the modeling capabilities of the Cordis-Anima system [8] [11]. In the sequel, we will make the assumption that the data $G(M), G(T)$ and $S P$ are known beforehand, and we will focus on the physical aspects of the motion generation problem.

\section{World modeling using physical models}

\subsection{Physical models}

Geometric models have been designed for representing shape properties and for defining macroscopic behaviours for solid objects. But, such models do not includes the ability of processing complex physical interactions, because the associated effects result from the integration of differential equations involving distributed force and position parameters. This is why researchers in the field of computer graphics and of man/machine communication have developed the concept of "physical model". The basic idea is to consider that any object motion and/or deformation is linked by physical laws to a set of forces whose application points depend on the intrinsic 
structure of the involved objects. A practical way for modelizing this phenomenon is to appropriately discretize the object and to characterize each obtained component by differential equations combining two dual variables: the force $F$ and the position $P$ (or the velocity $V$ ). Such a type of model is usually called "continuous lumped constant model". It basically permits the construction of complex objects by assembling object components using various connectors, for instance: connectors representing the non-linearities associated to the object boundaries (for modeling contact behaviours), or connectors defining some structural modifications (plasticity properties) [8] [11].

In our model, any object is represented by a structured set of spherical components (balls), and any pair of interacting components is connected by an appropriate connector. This approach allows us to process physical interactions using some elementary dynamic equations involving punctual masses only: a ball is seen as a pair $(m, r)$, where $m$ is an "active" punctual mass located at the center of the ball and $r$ is the radius of the associated interaction area (this area is generally characterized by a non-penetration law) [8]. Consequently, the physical model $\Phi(W)$ of a set $W$ of interacting objects can be represented by a network in which each node $n_{i} \in \mathcal{N}$ defines an ob ject component (a ball), and each arc $a_{i j} \in \mathcal{A}$ represents a physical interaction. $A$ node $n_{i}$ is characterized by a pair $\left(p_{i}, m_{i}\right)$, where $p_{i}$ and $m_{i}$ are respectively the position and the weight of the associated punctual mass; an arc $a_{i j}$ defines a particular interaction equation of the type $F_{i}=-F_{j}=\phi_{i j}\left(p_{i}, p_{j}\right)$, where $\phi_{i j}$ represents a linear damper/spring law or a particular non-linear interaction law.

\subsection{Terrain and vehicle modeling}

As explained in \&2, the geometric models $G(T)$ and $G(M)$ have to be converted into a physical representation $\Phi(T, M)$. The first step of the algorithm is to construct $\Phi(T)$ and $\Phi(M)$ using appropriate connected sets of balls.

Let $G(T)$ be a numerical model of the surface of $T$. Converting $G(T)$ into $\Phi(T)$ consists in finding an appropriate agglomerate of balls, in which each ball represents a punctual mass and its associated non-penetrating area -this area is characterized by an elastic/viscous repulsive law-. In our system, this agglomerate is constructed using a dynamic ball meshing algorithm consisting in "throwing" a set of interactive balls towards a rigid net representing the surface of $T$ [12], figute 2a illustrates. The sizes of the balls which are used in this algorithm depends on the spatial bandwith of $T$ and on the characteristics of $M$ (size of the wheels for instance). The interaction laws to use for completing $\Phi(T)$ are selected according to the physical characteristics of the ground. Until now, most of the experiments have been processed using a rigid ground (see \&6).

$G(M)$ includes shape and kinematics representations. This means that two main steps have to be applied for constructing $\Phi(M)$ : (1) selecting a representative set of punctual masses, and (2) connecting theses punctual masses in order to define rigid components and articulated mecanisms. Rigid components are modelized using almost indeformable spring connectors; articulated mecanisms are constructed using appropriate damper/spring based structures (see figure $2 \mathrm{~b}$ ).

The last step of the physical modeling process is to combine $\Phi(T)$ and $\Phi(M)$ in order to explicitly represent the vehicle/terrain interactions. This is done by adding new connectors between some pairs of punctual masses belonging to $\Phi(T) X \Phi(M)$. The interaction laws to associate to these connectors depends on both the physical characteristics of the ground and the vehicle locomotion mechanisms (wheeled vehicle, tracked vehicle ...). An implementation of a wheeled vehicle moving on a rigid ground is described in \&6. 
a)

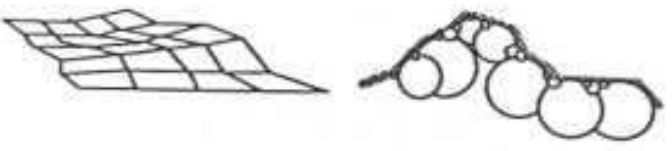

b)

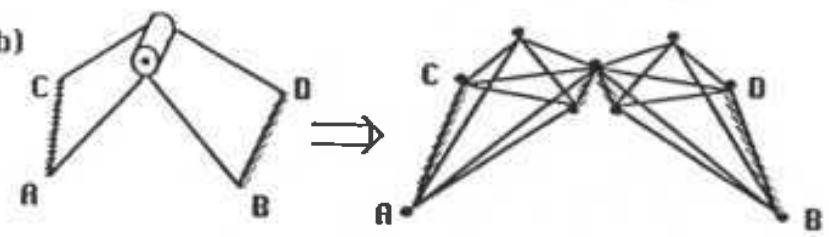

Figure 2: (a) Numerical model and physical model of $T$. (b) Modeling an articulated mechanism c)

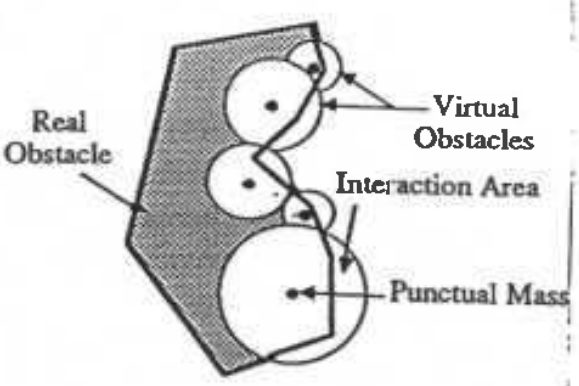

(c) A "generalized obstacle".

\section{Motion generation}

Let $W$ be a set of interacting objects and $F$ be an external force which is applied to any component of $\Phi(W)$. The dynamic behaviour of $W$ after having applied $F$ can be simulated at a given frequency using the following iterative algorithm:

1. Compute the forces to associate to each arc $a_{i j}$ in $\Phi(W)$ by evaluating the interaction function $\phi_{i j}$.

2. Compute the new position $p_{i}$ to associate to each node $n_{i}$ in $\Phi(W)$ using the Newtonian law:

$$
\sum_{\forall a_{i k}} F_{k}=m_{i} \cdot \gamma_{i}=m_{i} \cdot\left(d^{2} p_{i} / d t^{2}\right) \text {. }
$$

The same algorithm can be used for generating the motions of the vehicle $M$ by associating a propeling force $F$ to $M$. Since $M$ has to take into account the strategic information contained in $S P$ (see \&2), $F$ must be chosen in such a way that $M$ will move towards the next subgoal $g_{i}$ while following the channel $C$ and reacting with $T$. Two basic physical constructions have been developed for maping these motion constraints onto $\Phi(T, M)$ :

1. The generalized obstacles: The first motion constraint to satisfy is to force $M$ to follow $C$ while reacting with $T$. This means that both $\Phi(T)$ and $\Phi(C)$ have to be used for physically guiding $M$. In other words, $\Phi(T)$ and $\Phi(C)$ have to be combined within an unified model $\Phi(T \oplus C)$ refered as the "generalized obstacle model". A practical way for implementing this capability is to consider that $C$ is delimited by a set $\left\{O_{1}, O_{2} \ldots O_{m}\right\}$ of real or fictitious obstacles (see figure 1). Then, each $O_{i}$ can be converted into a model $\Phi\left(O_{i}\right)$ which can be easily combined with $\Phi(T)$ : both $\Phi\left(O_{i}\right)$ et $\Phi(T)$ are made up of agglomerates of balls. The main difference between $\Phi\left(O_{i}\right)$ and $\Phi(T)$ relies on the fact that the interaction areas which are associated to the balls belonging to $\Phi\left(O_{i}\right)$ include a safety distance preventing $M$ to collide with $O_{i}$ (see figure 2c). Using this approach, it becomes possible to simultaneously solve the locomotion problem and the obstacle avoidance problem (see \& $\&$ ).

2. The physical targets: The second motion constraint to satisfy is to force $M$ to move towards the next subgoal $g_{i}$. A practical way for implementing this capability is to represent $g_{i}$ by an attractive punctual mass $\Phi\left(g_{i}\right)$ called "physical target". The basic interaction law which is associated to such an entity is an attractive non-linear viscous/elastic law. Some other interaction laws may also be used for implementing more complex vehicle behaviours; moreover, sophisticated motion strategies may be obtained in a similar way by combining the effects of 
several physical targets. Thus, it becomes possible to constantly generate an appropriate propeling force $F$ for $M$ by only evaluating the current state of the target/vehicle interaction (see \&6). Physical targets may also be interactively moved in the scene for the purpose of locally modifying the motion commands to apply to $M$. In the Cordis-Anima working environment, this can be done using a real-time physical simulator and a gestural retroactive device previously developed by the Acroe-Lifia team (see \& 6). Such a capability is potentially usefull for interactively generating appropriate manoeuvres in intricate situations - because force feedback may help the operator-, but it is not clear (at the present time) how the resulting physical data can be converted into a strategic geometric information for the planner.

\section{An extention of the "Predictive Display"}

The new approach we have presented in this paper can be placed within the framework of an extention of the "Predictive Display" concept. The objective is to extend the task performance visualisation functions by offering to the robotic operator a complete reconstruction of the natural piloting situation. This can be done by combining a motion generator based on dynamic models (see \&4) and gestural devices for providing the operator with tactile feedback.

The whole system made of the modeler-simulator for physical object and of force feedback gestural devices allow, by mean of manipulation of physical targets for example, a genuine gestural relationship with the simulated environement (see figure 3 ).

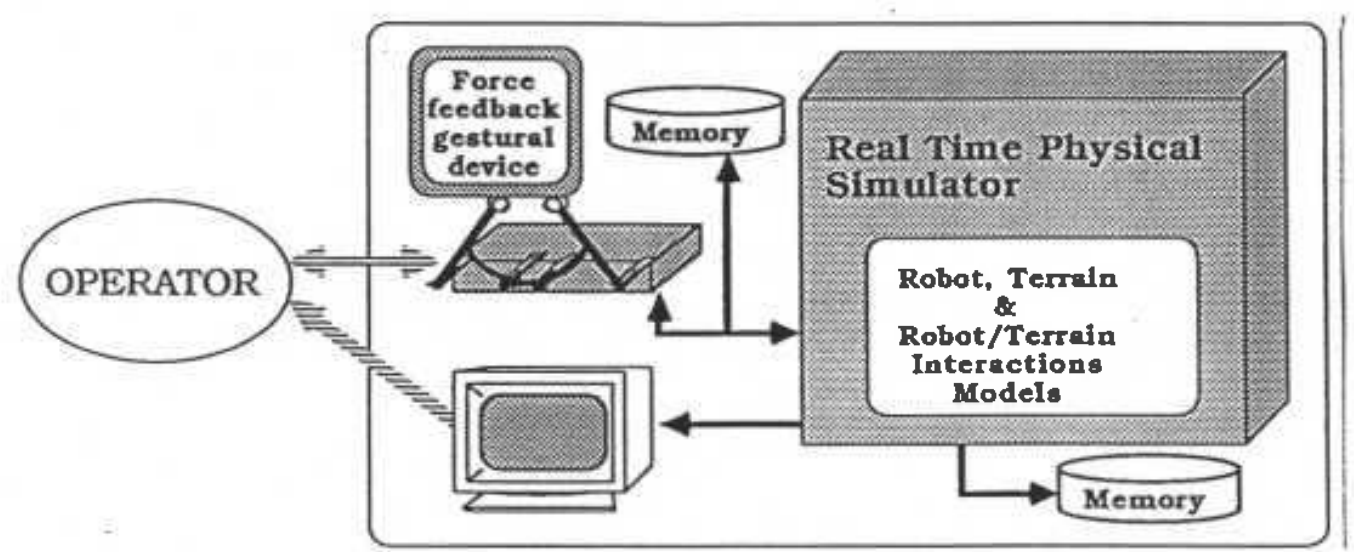

Figure 3: Reconstruction of the natural piloting situation.

The function of the gestural transducer is to liaise the relation between the human operator gesture and the numeric phenomenon produced by the computer. The gestural relationship is typically bi-directionnal, and this kind of device must fulfil two main functions:

1. to pick up the characteristic information about the control gesture.

2. to set a mechanical resistance against the operator gesture according to the numerical signal from the simulation process. This function is fundamental since it provides the user with an interface allowing an efficient control of the simulated system.

From 1978, the ACROE research team has introduced the idea of a gestural man-machine communication with "tactile feedback" in the framework of sound synthesis, and more generally of man machine communication problems [3] [4]. The studies carried out during the ten past 
years has led to the caracterisation and to the design of the requisite device, which has been called Modular Force Feedback Transducer (MFFT). An international patent covers both its modularity principle and its motor technology, that was specially developed to achieve the MFFT's performances [2].

Two typical situations constitute the reference to determine the gestural device characteristics. We want to have the ability to restitute good buffers, which correspond with collisions against hard surfaces. We also want to be able to restitute texture sensation in a general sense. Viscous and dry frictions are involved in these surface contacts. The sensor and specially the motors performances can be deduced from these situations and from the human gesture characteristics. For example the motor performance entails ultra-rapid, and accurate "response", (in some cases the bandwidth of the mechanical phenomena concerned in the gesture can go as far as 700 or $800 \mathrm{~Hz}$ ) and sizable power (up to pulse of several tenths of $\mathrm{KgF}$ to simulate rigid obstacles).

Moreover, it is not foreseeably possible to design a transducer for the gestural channel as general as a loud speaker is to the acoustic channel or a graphic screen to the visual channel.

This remark therefore induced the ACROE researchers to investigate what kind of device would guarantee a relative variety of gesture. The complete device comes in the form of an extremely compact unit built from sixteen sliced "motor/sensor modules". Every slice has a thickness of $13.75 \mathrm{~mm}$ and offer a one DDL gestural channel. The sixteen motor/sensor unit is organized according to a linear placing of the sliced motors (figure 4).

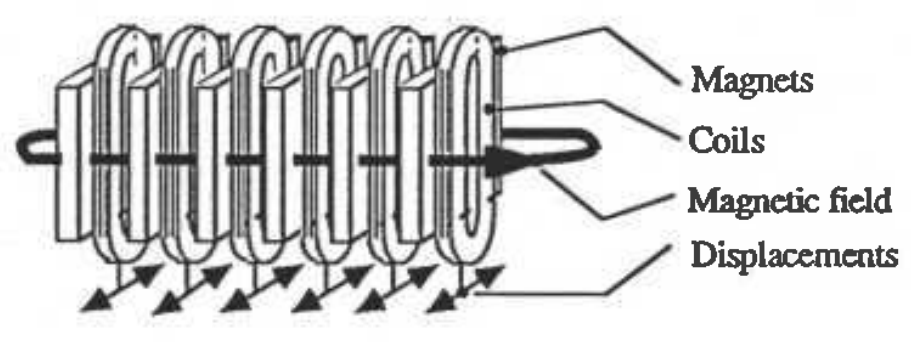

The sliced motor block

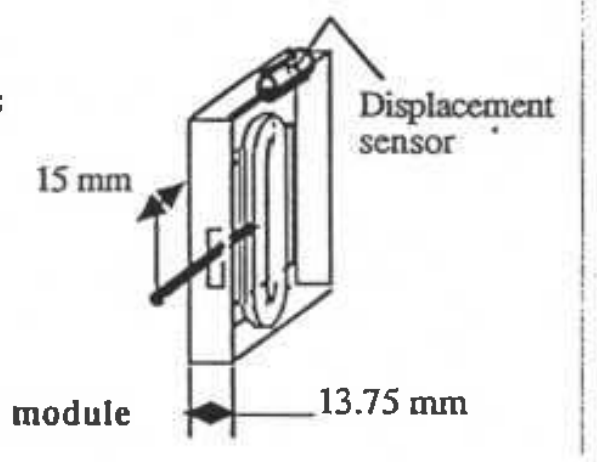

Figure 4: The sliced motor block and the motor/sensor module.

Motor/sensor module characteristics:

Geometric:

- mobile motor coil stroke

- Slice thickness

Sensor:

- position sensor LVDT, res.
$15 \mathrm{~mm}$

$13.75 \mathrm{~mm}$

approx. $3 \mu \mathrm{m}$
Motor:

- flat copper mobile coils vacuum soaked,

- rare carth / cobalt magness. effective induction

$0.65 \mathrm{~T}$

- mobile coil mass

$200 \mathrm{gr}$

- friction

- Maximal permanent force $<9.10-3 \mathrm{~N}$

- Maximal transitory force $40 \mathrm{~N}$

$80 \mathrm{~N}$

- Maximal acceleration off-load $\quad 660 \mathrm{~m} / \mathrm{s} 2$

- Response delay to the input control, (movement blocked) 
The effective morphology is determined by the association of a certain number of motor/sensor modules from the sixteen DDL unit and by the "Covering" that is given to the
whole.

This "Covering" consists of a very simple and strongly built mechanical device chosen from an "assortment" of various possibilies which is mounted onto the motor/sensor module to combine.

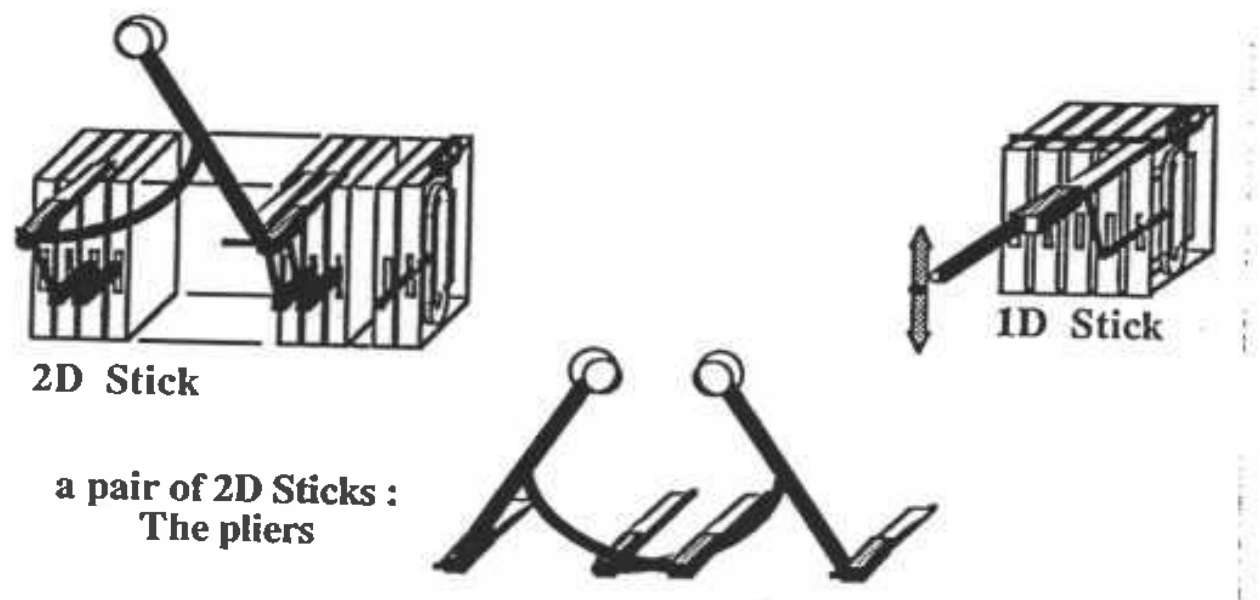

Figure 5: 1D and 2D joysticks. A mechanically connected transmition arm allows us to use one module as a 1D device or to combine two independant motor/sensor modules to create a system with two degrees of liberty.

It is therefore possible from this principle to define a great number of interchangeable covering like 1D, 2D and 3D covering (figure 5). For example in order to manipulate a $3 \mathrm{D}$ solid, we need a six DDL device which will be obtained from the association of six motor/sensor modules.

\section{Implementation and experiments}

\section{Implementation}

The approach described in this paper has been implemented and tested using the Cordis-Anima modeler/simulator [11]. The working environment of this system includes a VAX 750, an array processor AP120, an Evans \& Sutherland PS350 graphic workstation, and a gestural retroactive device. It provides the user with real-time simulation capabilities and with multi-modal communication tools. Thus, complete information concerning the simulated physical process (animated graphic simulation, force feedback and states of the dynamic parameters) can be used by the operator for evaluating the quality of the studied motion plan. The geometric models of $T$ and $M$, and the proposed motion plans are initially constructed using our CAD-Robotics system (the ACT system) working on a Silicon Graphics workstation. The figure 6 shows a visualization of a scene which has been described using the geometric models of an articulated rover and of a Marsian landscape. As previously mentionned, no complete connection has been inplemented yet between the two previous systems. Consequently, the required physical models are currently constructed by the operator (within the Cordis-Anima environment) from a description of the outputs of the ACT system.

Experiments involving several types of vehicle (tracked vehicle, wheeled vehicle, articulated vehicle), several types of terrain, and several types of vehicle/terrain interaction have been successfully processed. Some sequences of motions which have been generated by the system for avoiding a set of obstacles and for moving a rover on a solid hilly terrain are presented below. 


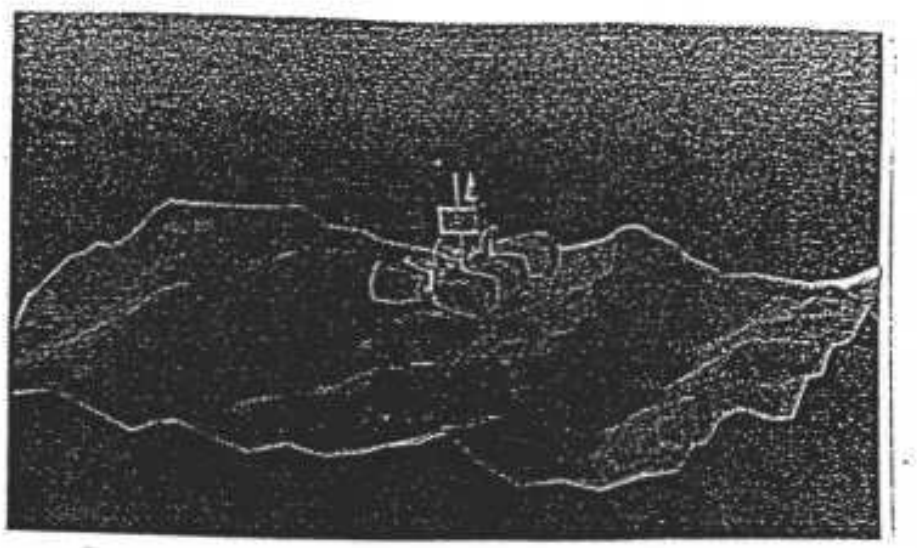

Figure 6: An articulated rover in a natural environment.
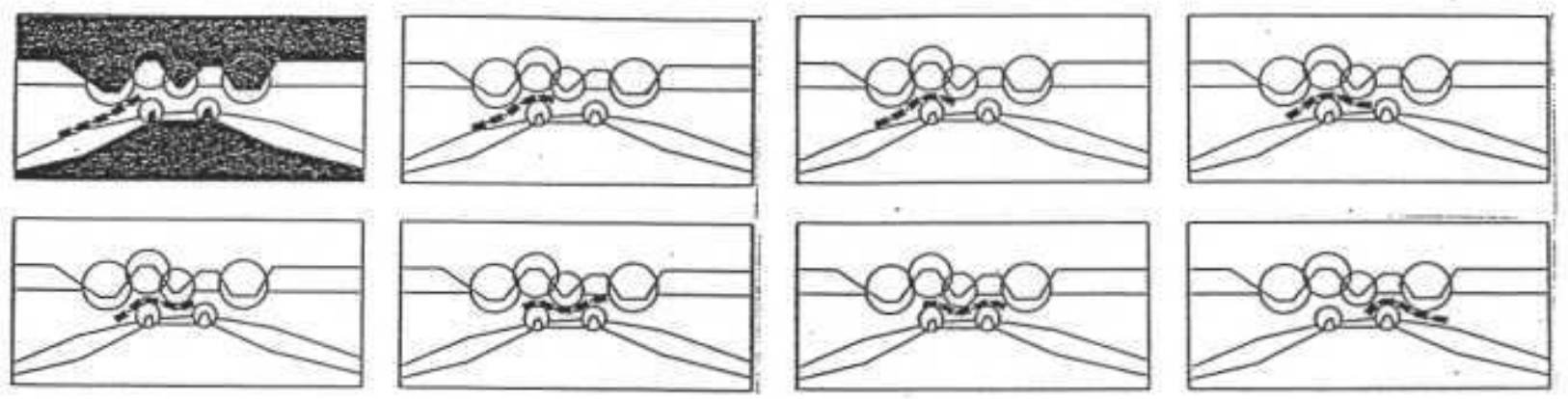

Figure 7: Avoiding 2D obstacles with a train-like vehicle.

\section{Obstacle avoidance}

In this experiment, the vehicle is a train-like mobile moving in a planar environment containing a set of obstacles to avoid (see figure 7). These obstacles are represented in the picture using the grey color, and their associated generalized obstacles are represented by white circular regions. Only the vehicle/obstacle interactions have been considered in this experiment. These interactions have been modelized using a classical repulsive viscous/elastic law including some non-linearities (instantaneous changes between contact and non-contact states).

The movements of the vehicle are generated here using an attractive physical target whose position is interactively specified by the operator. The resulting motion will make the vehicle to move through the narrow corridor only if the target has been correctly positionned. The solution shown in the figure 7 has been easily found after a few attempts performed in the simulated environment (i.e. in a few seconds).

\section{Dynamic locomotion on a hilly terrain}

In this experiment, the vehicle is a $3 \mathrm{D}$ wheeled vehicle having several passive joints and six independant conical wheels (see figure 8 ). The purpose of the articulated mechanical structure is to provide the rover with the capability of continuously maintaining a sufficient number of contact points between the wheels and the ground when getting over some natural obstacles (rocks, small hills and hollows ...). This is achieved by automatically modifying the configuration of the articulated mechanical structure using gravity and reaction forces produced by the wheels/ground interactions.

The terrain which has been used for this experiment has been modelized using an almost rigid agglomerate of balls having non-penetrating properties (see \& 3 ). The vehicle has been 

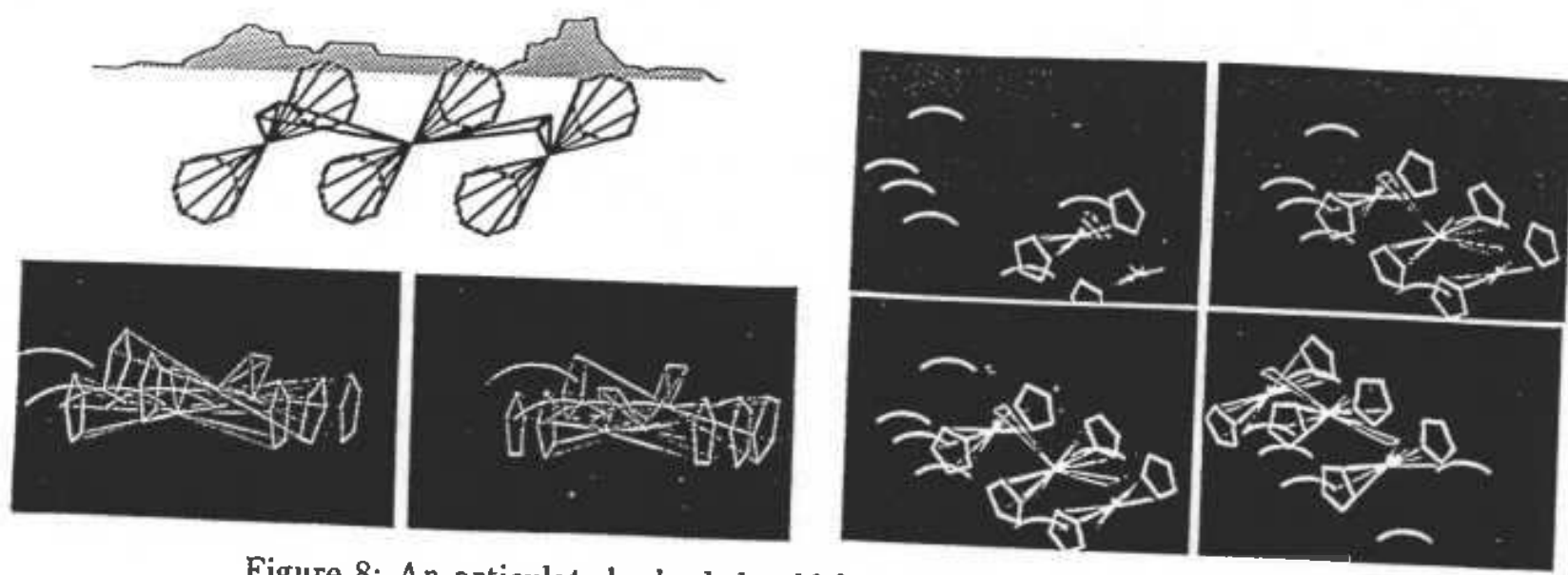

Figure 8: An articulated wheeled vehicle moving on a solid hilly terrain.

modelized as explained in \&3. Only the punctual masses which has been associated to the wheels are considered when computing the vehicle/terrain interactions.

Two basic interaction models have been combined for providing the rover with the required dynamic behaviour: a classical non-penetrating model and a model aimed at generating slidingfree rolling motions (this model combines a friction term with the instantaneous tangential speed is applied to the rover is which is involved in the current contact). The propeling force which shown in the figure 8 .

\section{Conclusion}

We have presented a method for solving the problem of planning the motions of a complex land vehicle moving in a natural environment containing both obstacles that have to be avoided and obstacles that can be crossed over by the vehicle. The main contribution of the paper is a motion generator which predicts the dynamic behaviour of the vehicle when executing a given nominal motion plan. For that purpose, we have shown how the dynamic behaviour of such robots could be modelized and simulated using the concept of "physical model" which had initially been developed to solve computer graphics and man/machine communication problems. We have also shown how strategic and tactical information concerning the motion to execute could be modelized an processed using this type of model. Several nice experimental results obtained using the Cordis-Anima modeler/simulator have finally be described.

The current work is oriented towards two main directions: completing the connection between the path planner (strategic layer) and the motion generator (tactical layer), and developing improved techniques for constructing more complete terrain and vehicle physical models.

\section{Acknowledgement}

The research carried out by the Acroe/Lifia team is mainly supported by the French Ministère de la Culture. The work presented in this paper las been partly supported by the CNES (French spatial agency) through the RISP national project. It also has been partly supported by the Rhonc-Alpes Region through the IMAG/INRIA Robotics project SHARP. 


\section{References}

[1] J.Barraquand and J.C.Latombe: "On non holonomic mobile robots and optimal maneuvering”, Revue d'Intelligence Artificielle, Vol.3, Nb.2, 1989, pp.77-103.

[2] Brevet INPI - N. 88 14064, déposé le 13 Octobre 1988 - titre: “Clavier Retroactif Modulaire et actionneur modulaire extra-plat". Inventeurs : C. Cadoz, L. Lisowski, J.L.Florens.

[3] C.Cadoz, J.L.Florens, A. Luciani: "Responsive Input Devices and sound synthesis by simulation of instrumental mechanisms : The CORDIS system ", Computer Music Journal, 8 N. 3, pp. 60-73.

[4] C.Cadoz, L.Lisowski, J.L.Florens: “A modular feedback keyboard design", Computer Music Journal, Vol.14, Nb.2, MIT Press, 1990, pp.47-51.

[6] T.Fraichard, C.Laugier, G.Lievin: "Robot motion planning: the case of non holonomic mobiles in a dynamic world", IROS'90, Tsuchiura, July 1990.

[7] T.Fraichard and C.Laugier: "On line reactive planning for a non holonomic mobile in a dynamic world", IEEE International Conference on Robotics and Automation, Sacramento, April 1991.

[8] S.Jimenez: “Modélisation et simulation d'objets volumiques déformables complexes”, DEA Technical Report, INPG, June 1989 (in French).

[9] C.Laugier: "Geometric reasoning in motion planning", Technical Report no.932, INRIA, December 1988.

[10] J.P.Laumond, M.Taix, P.Jacobs: “A motion planner for car-like robots based on a mixed global/local approach", IROS'90, Tsuchiura, July 1990.

[11] A.Luciani and al: "An unified view of multiple behaviour, flexibility, plasticity and fractures: balls, bubbles and agglomerates", IFIP WG 5.10 on Modeling in Computer Graphics, Springer Verlag, 1991, pp.55-74.

[12] J.Moutard: "Planification et contrôle d'exécution simulé d'un véhicule autonome planétaire", DEA Technical Report, INPG, June 1991 (in French).

[13] M.Raibert: “Dynamic legged robots”, ISER'88, Sydney, November 1988. 